\title{
Intraluminal anatomy of the transverse sinus: implications for endovascular therapy
}

\author{
Juan J. Altafulla ${ }^{1}$, Joshua Prickett ${ }^{2}$, Joe Iwanaga ${ }^{3,4}$, Aaron S. Dumont ${ }^{3}$, R. Shane Tubbs ${ }^{3,5,6,7}$ \\ ${ }^{1}$ Department of Neurosurgery, Hospital Santo Tomas, Panamá, Panama, ${ }^{2}$ Aiken Regional Medical Centers, Aiken, SC, ${ }^{3}$ Department of Neurosurgery, \\ Tulane Center for Clinical Neurosciences, Tulane University School of Medicine, New Orleans, LA, USA, ${ }^{4}$ Division of Gross and Clinical Anatomy, \\ Department of Anatomy, Kurume University School of Medicine, Kurume, Japan, ${ }^{5}$ Department of Anatomical Sciences, St. George's University, St. \\ George's, Grenada, ${ }^{6}$ Department of Neurosurgery and Ochsner Neuroscience Institute, Ochsner Health System, New Orleans, LA, ${ }^{7}$ Department of \\ Structural and Cellular Biology, Tulane University School of Medicine, New Orleans, LA, USA
}

\begin{abstract}
Trabeculae or septations in the transverse sinus can have potentially life-threatening clinical significance. The current study demonstrates trabecula/septum patterning within the transverse sinus with measurements and distribution data supplemented by imaging, and describes the possible etiology of idiopathic intracranial hypertension and turbulent blood flow in the transverse sinus. Twenty-four sides from 12 cadaveric heads, all fresh-frozen, were used (five males, seven females; age at death 65-91 years, mean 79.1 years). The length and diameter of the transverse sinus were measured along with the number and locations of septations/trabeculae and their tensile strength. The mean length of the transverse sinus was $68.43 \mathrm{~mm}$ on the right side and $74.31 \mathrm{~mm}$ on the left. A total of 42 septations were found in the 24 transverse sinuses. The number of septations per side ranged from zero to four with a mean of 1.75 . The septations were located in the proximal $1 / 3$ in $54.8 \%$ (23/42), the middle $1 / 3$ in $21.4 \%$ (9/42), and the distal $1 / 3$ in $23.8 \%$ (10/42). The work presented here furthers our understanding of transverse sinus anatomy, including its detailed internal architecture. The measurements can provide a technical guide for neurosurgeons and influence instrument selection when a large thrombus forms or anchors in one of these trabeculae or septa and necessitates treatment.
\end{abstract}

Key words: Transverse sinus, Septations, Trabeculae, Idiopathic intracranial hypertension, Papilledema

Received February 26, 2020; 1st Revised April 1, 2020; 2nd Revised April 22, 2020; Accepted April 27, 2020

\section{Introduction}

Among the dural venous sinuses that function as a web of venous passages for draining blood from the brain, the right and left transverse sinuses exclusively drain blood arising from the superficial and deep aspects of the brain, respectively. The right transverse sinus is typically larger than the left because it drains most of the blood from superficial

\section{Corresponding author:}

Joe Iwanaga (iD

Department of Neurosurgery, Tulane Center for Clinical Neurosciences, Tulane University School of Medicine, New Orleans, LA 70112, USA E-mail: iwanagajoeca@gmail.com portions of the brain. The transverse sinus originates in the posterior cranial fossa at the junction of the superior sagittal, straight, and occipital sinuses, termed the 'confluence of sinuses' or torcular Herophili $[1,2]$.

Cases of trabeculae or septations in the transverse sinus have been observed with potentially life-threatening clinical significance. Strydom et al. [3] reported that $29.4 \%$ of 102 cadavers and patients presented with such trabeculae or septa and concluded that these entailed a potential for defects in venous filling, further indicating the role of transverse sinus anatomy in idiopathic intracranial hypertension (IIH). Subramaniam et al. [4] found that septations in the transverse sinus could affect blood drainage, causing turbulence and therefore potentially leading to thrombosis or thromboembolism.

\section{Copyright ( 2020 . Anatomy \& Cell Biology}

This is an Open Access article distributed under the terms of the Creative Commons Attribution Non-Commercial License (http://creativecommons.org/licenses/by-nc/4.0/) which permits unrestricted non-commercial use, distribution, and reproduction in any medium, provided the original work is properly cited. 
The present paper describes trabecular and septational patterning in the transverse sinus with measurements and distribution supplemented by imaging, and describes a potential etiology of IIH and turbulent blood flow in the transverse sinus.

\section{Materials and Methods}

The anatomical quality assurance checklist was used for this study [5]. Twenty-four sides from eleven Caucasian and one Asian cadaveric heads, all fresh-frozen, were used (five males and seven females, age at death 65-91 years, mean 79.1). The specimens were placed in supine position, a circumferential incision was made $1 \mathrm{~cm}$ above the orbital margin, and a craniotomy was performed using an electric bone saw (Stryker Inc., Kalamazoo, MI, USA). All the supratentorial neural structures were dissected and removed to expose the entire transverse sinus, the length and diameter of which were recorded using the bifurcation at the level of the torcular Herophili as the origin and the curvature at the transverse-sigmoid sinus junction as it entered the petrous bone as the terminus. The transverse sinus was then transected entirely to expose its inner architecture. The number, locations, tensile strength, and positions of the septations inside the sinus were recorded. All measurements were made with microcalipers (Mitutoyo Co., Kanagawa, Japan) and a tensometer (M2-200; MARK10 Co., Copiague, NY, USA). Statistical analysis between sides and sex were performed using Student t-tests with significance set at $P<0.05$.

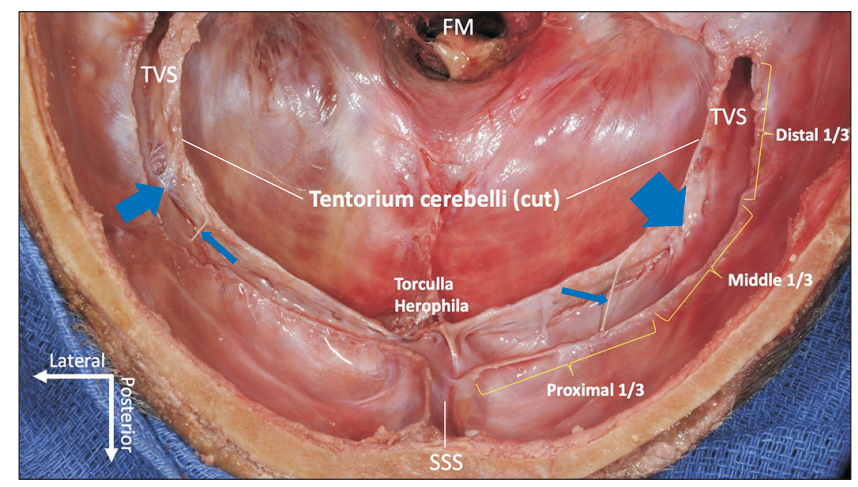

Fig. 1. Right transverse sinus dissected in its entirety. Septations are seen in the middle $1 / 3$ and distal $1 / 3$ (blue arrows). FM, foramen magnum; SSS, superior sagittal sinus; TVS, transverse sinus.

\section{Results}

Transverse sinus septations were defined as intraluminal trabeculations arising from either the lateral or the medial aspect of the sinus wall and transecting the space. For better understanding, the sinus was divided into three parts (proximal, medial, and distal), distinguished by the points at which it changes direction (Fig. 1).

\section{Measurements of transverse sinuses}

The mean length of the transverse sinus was $68.4 \pm 16.5$ $\mathrm{mm}$ (range, $19.5-80.4 \mathrm{~mm}$ ) on the right side and $74.3 \pm 4.6$ $\mathrm{mm}$ (range, $66.02-80.2 \mathrm{~mm})$ on the left $(P=0.18)$. There were no significant sex $(P=0.21)$ or side differences $(P=0.18)$ regarding the length.

The mean diameter was $6.2 \pm 1.3 \mathrm{~mm}$ (range, $2.5-7.6 \mathrm{~mm}$ ) on right sides and $6.3 \pm 0.7 \mathrm{~mm}$ (range, $5.3-7.3 \mathrm{~mm}$ ) on left sides. There were no significant sex $(P=0.13)$ or side differences $(P=0.78)$ regarding diameter.

One specimen, a 75-year-old female Caucasian, presented with a hypoplastic right transverse sinus that measured 19.5 $\mathrm{mm}$ in length and $2.5 \mathrm{~mm}$ in diameter (less than half that of the other specimens).

\section{Septations within the transverse sinuses}

A total of 42 septations were found in the 24 transverse sinuses studied. The number of septations per side was one in $29.2 \%$ (7/24), two in $33.3 \%(8 / 24)$, three in $20.8 \%(5 / 24)$, four in $4.2 \%(1 / 24)$, and none in $12.5 \%(3 / 24)$, with a mean of 1.75 (Fig. 2). One specimen presented no septation in either the left or right transverse sinus; one had none on the right side (hypotrophic) but three on the left.

The septations were most commonly located where the

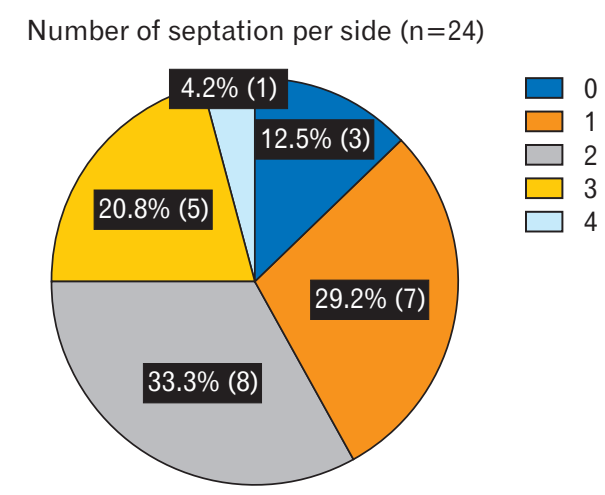

Fig. 2. Number of septations in the transverse sinus per side. 


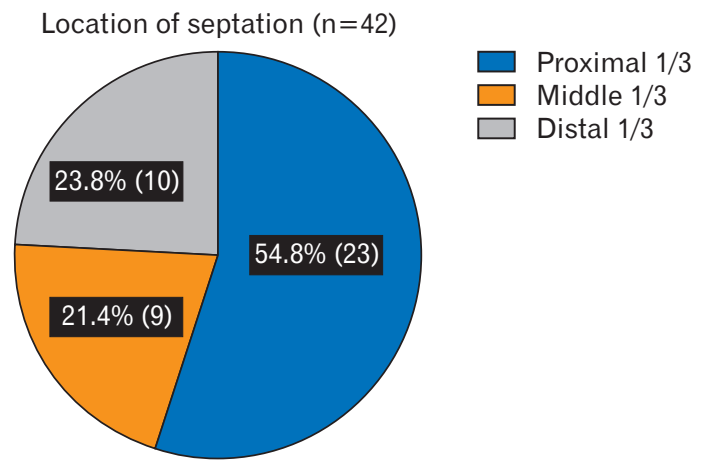

Fig. 3. Location of septations within the transverse sinuses.

sinus changed direction. Most of them were at either end of the transverse sinus: $54.8 \%$ (23/42) in the proximal 1/3, $21.4 \%$ (9/42) in the middle $1 / 3$, and $23.8 \%(10 / 42)$ in the distal $1 / 3$ (Fig. 3).

The mean tensile strength of the septations was $7.8 \pm 2.4$ $\mathrm{N}$ (range, 4-11 N) for the right transverse sinus and 6.3 \pm 2.7 $\mathrm{N}$ (range, 3-11 N) for the left. There were no significant sex ( $P=0.78)$ or side differences $(P=0.21)$ in regard to the tensile strength.

\section{Discussion}

The cerebral venous system is far more variable than the cerebral arterial system, differing not only among individuals but also between hemispheres in the same brain. This makes it difficult to classify the cerebral veins $[6,7]$. In contrast to the arterial system, the venous drainage of the brain is only partly understood. Most authors agree that it can be divided, according to its relationship to the cortex, into dural venous sinuses (superficial circulation) and deep cerebral veins (deep circulation) [8].

The dural venous sinuses are important for regulating normal venous drainage. Small changes in posture can translate into significant changes in blood flow, redirecting drainage through the internal jugular vein or the vertebral veins $[9,10]$. Postural change is a key regulator of blood flow in the pathological brain, as in cases of traumatic brain injury, IIH, subarachnoid hemorrhage, and stroke, serving not only as bedside assessment but also as a therapeutic option [11].

The right transverse sinus is usually larger than the left and asymmetry of the transverse sinuses is not uncommon [12]. Unilateral hypoplasia or aplasia, which was also found in the present study, can be observed in $20 \%$ to $39 \%$ of cases [12-14]. In this study, the mean diameter of the transverse sinus was $6.21 \mathrm{~mm}$ and $6.30 \mathrm{~mm}$ on right and left sides, respectively. Thus, the morphology of the transverse sinus is variable.

Subramaniam et al. [4] investigated the relationship between transverse sinus stenosis and internal septations, providing a solid basis for a hypothesis about IIH and presenting results similar to ours, though not all variables (such as tensile strength and specific locations of the septations within the sinus) were recorded [4]. Other studies examining the variability of sinus wall thickness across its course showed it to be thickest at the level of the torcular Herophili and thinnest at the level of the superior jugular bulb and sigmoid sinus [15].

To our knowledge, this is the first study to describe the architectural components inside the transverse sinus in detail, including the tensile strength of the trabeculae and septations. Furthermore, measurements of the diameter and length of the sinus could elucidate the difference between intracranial and dural venous pressures, which is important in venous collapse in both normal and pathological states [16]. This new evidence provides a better anatomical and physiological understanding of why and where progressive collapse of the transverse sinus occurs secondary to increased intracranial pressure [17].

\section{Implications in intracranial hypertension states and endovascular pathology}

The transverse and sigmoid sinuses are known sites for endovascular stent placement for treating IIH, sinus stenosis and sinus thrombosis. During a progressive increase of intracranial pressure the transverse sinus undergoes progressive collapse. The tensile strength values we obtained for the inner septations of the transverse sinus suggest that these septations help to stabilize and maintain the three-dimensional structure of the sinus where the need for support is greatest, i.e., in areas of high hemodynamic stress such the locations of highest curvature.

Our diameter and length measurements are consistent with those reported in the literature and could easily be translated into estimating turbulent versus laminar flow in the sinus when complemented with blood pressure and hematocrit values [18]. These measurements are particularly useful during endovascular procedures where a stent is placed in the sinus lumen as a treatment option for IIH [1921]. Our findings could help in thrombectomy planning, since a thrombus formed within one of the large trabeculae 
or septations would not resolve completely with conventional thrombolysis and could lead to infarction [4].

In conclusion, the work presented here furthers our understanding of transverse sinus anatomy, including its detailed internal architecture. The measurements in the current study could serve as a technical guide for neurosurgeons, and could influence instrument selection when a large thrombus is formed or attached to one of these trabeculae or septa and requires treatment. Further clinical studies should examine a possible connection between the intravascular septae of the transverse sinus and sinus thrombosis formation or IIH.

\section{ORCID}

Juan J. Altafulla: https://orcid.org/0000-0002-2110-8665

Joshua Prickett: https://orcid.org/0000-0002-0716-7554

Joe Iwanaga: https://orcid.org/0000-0002-8502-7952

Aaron S. Dumont: https://orcid.org/0000-0002-8077-8992

R. Shane Tubbs: https://orcid.org/0000-0003-1317-1047

\section{Author Contributions}

Conceptualization: JI, ASD, RST. Data acquisition: JJA, JI, RST. Data analysis or interpretation: JJA, JP. Drafting of the manuscript: JJA, JP. Critical revision of the manuscript: JI, ASD, RST. Approval of the final version of the manuscript: all authors.

\section{Conflicts of Interest}

No potential conflict of interest relevant to this article was reported.

\section{References}

1. Standring S. Gray's anatomy: the anatomical basis of clinical practice. 40th ed. Edinburgh: Churchill Livingstone/Elsevier, 2008.

2. Curé JK, Van Tassel P, Smith MT. Normal and variant anatomy of the dural venous sinuses. Semin Ultrasound CT MR 1994;15:499-519.

3. Strydom MA, Briers N, Bosman MC, Steyn S. The anatomical basis of venographic filling defects of the transverse sinus. Clin Anat 2010;23:153-9.

4. Subramaniam RM, Tress BM, King JO, Eizenberg N, Mitchell PJ. Transverse sinus septum: a new aetiology of idiopathic intracranial hypertension? Australas Radiol 2004;48:114-6.
5. Tomaszewski KA, Henry BM, Kumar Ramakrishnan P, Roy J, Vikse J, Loukas M, Tubbs RS, Walocha JA. Development of the Anatomical Quality Assurance (AQUA) checklist: guidelines for reporting original anatomical studies. Clin Anat 2017;30:1420.

6. Lake S, Altafulla JJ, Iwanaga J, Oskouian RJ, Loukas M, Tubbs RS. A cadaveric case of a circular torcular Herophili. Cureus 2018;10:e3099.

7. Ahmed MS, Imtiaz S, Shazlee MK, Ali M, Iqbal J, Usman R. Normal variations in cerebral venous anatomy and their potential pitfalls on 2D TOF MRV examination: results from a private tertiary care hospital in Karachi. J Pak Med Assoc 2018;68:1009-13.

8. Chandra A, Li WA, Stone CR, Geng X, Ding Y. The cerebral circulation and cerebrovascular disease I: anatomy. Brain Circ 2017;3:45-56.

9. Schaller B. Physiology of cerebral venous blood flow: from experimental data in animals to normal function in humans. Brain Res Brain Res Rev 2004;46:243-60.

10. Valdueza JM, von Münster T, Hoffman O, Schreiber S, Einhäupl KM. Postural dependency of the cerebral venous outflow. Lancet 2000;355:200-1.

11. Donnelly J, Budohoski KP, Smielewski P, Czosnyka M. Regulation of the cerebral circulation: bedside assessment and clinical implications. Crit Care 2016;20:129.

12. Alper F, Kantarci M, Dane S, Gumustekin K, Onbas O, Durur I. Importance of anatomical asymmetries of transverse sinuses: an MR venographic study. Cerebrovasc Dis 2004;18:236-9.

13. Fofi L, Giugni E, Vadalà R, Vanacore N, Aurilia C, Egeo G, Pierallini A, Barbanti P. Cerebral transverse sinus morphology as detected by MR venography in patients with chronic migraine. Headache 2012;52:1254-61.

14. Han K, Chao AC, Chang FC, Hsu HY, Chung CP, Sheng WY, Chan L, Wu J, Hu HH. Diagnosis of transverse sinus hypoplasia in magnetic resonance venography: new insights based on magnetic resonance imaging in combined dataset of venous outflow impairment case-control studies: post hoc case-control study. Medicine (Baltimore) 2016;95:e2862.

15. Balik V, Uberall I, Sulla I, Ehrmann J, Kato Y, Sulla IJ, Takizawa K. Variability in wall thickness and related structures of major dural sinuses in posterior cranial fossa: a microscopic anatomical study and clinical implications. Oper Neurosurg (Hagerstown) 2019;17:88-96.

16. DE Simone R, Ranieri A, Bonavita V. Starling resistors, autoregulation of cerebral perfusion and the pathogenesis of idiopathic intracranial hypertension. Panminerva Med 2017;59:7689.

17. Shim EB, Heldt T. Fluid-structure interaction in the cerebral venous transverse sinus. Conf Proc IEEE Eng Med Biol Soc 2018;2018:45447.

18. Boddu SR, Gobin P, Oliveira C, Dinkin M, Patsalides A. Anatomic measurements of cerebral venous sinuses in idiopathic intracranial hypertension patients. PLoS One 2018;13:e0196275.

19. Cappuzzo JM, Hess RM, Morrison JF, Davies JM, Snyder KV, 
Levy EI, Siddiqui AH. Transverse venous stenting for the treatment of idiopathic intracranial hypertension, or pseudotumor cerebri. Neurosurg Focus 2018;45:E11.

20. Miyachi S, Hiramatsu R, Ohnishi H, Takahashi K, Kuroiwa T. Endovascular treatment of idiopathic intracranial hypertension with stenting of the transverse sinus stenosis. Neurointerven- tion 2018;13:138-43.

21. Patsalides A, Oliveira C, Wilcox J, Brown K, Grover K, Gobin YP, Dinkin MJ. Venous sinus stenting lowers the intracranial pressure in patients with idiopathic intracranial hypertension. J Neurointerv Surg 2019;11:175-78. 